\title{
Physics potential of Hyper-Kamiokande for neutrino oscillation measurements
}

\author{
Christophe Bronner*十 \\ Kamioka Observatory, Institute for Cosmic Ray Research, The University of Tokyo \\ E-mail: christophe.bronnereipmu.jp
}

In this paper, we present the potential of the next generation neutrino experiment HyperKamiokande for the study of neutrino oscillations. We first present the sensitivity of the experiment for the exclusion of the conservation of CP symmetry, and the determination of the mass hierarchy and of the octant of $\theta_{23}$ using a 187kton fiducial mass detector. We then discuss the improvements obtained with a second detector of identical fiducial mass in different configurations. With a single detector, and combining the analysis of accelerator neutrinos ( $295 \mathrm{~km}$ baseline) and atmospheric neutrinos, Hyper-Kamiokande can exclude the conservation of CP symmetry in neutrino oscillations with $5 \sigma$ (resp. $3 \sigma$ ) significance for $57 \%$ (resp. $74 \%$ ) of the possible true values of $\delta_{C P}$, and determine the mass hierarchy with more than $3 \sigma$ significance. This number increases to $4 \sigma$ significance if a second detector is added close to the first one in a staged approach, and to $6 \sigma$ if this additional detector is instead located in Korea, giving a longer baseline for the accelerator neutrinos.

The 19th International Workshop on Neutrinos from Accelerators-NUFACT2017

25-30 September, 2017

Uppsala University, Uppsala, Sweden

\footnotetext{
*Speaker.

${ }^{\dagger}$ On behalf of the Hyper-Kamiokande proto-collaboration
} 


\section{The Hyper-Kamiokande project}

Neutrino oscillations have been extensively studied since their discovery at the end of the 20th century, and the phenomenon is now well established after the observations of both the appearance and the disappearance of neutrinos of a certain flavor. Most of the parameters of the 3 flavor oscillation model have been measured, leaving three main open questions: whether neutrino oscillations respect $\mathrm{CP}$ symmetry, a symmetry between matter and anti-matter, what the ordering of the neutrino mass eigenstates is, and the octant of $\theta_{23}$. Those questions can be addressed by precise measurements of the values of some of the model parameters. However, those measurements correspond to the observation of more subtle variations in the oscillation pattern than what has been done so far, and will require both precise understanding of the systematic uncertainties, and large statistics.

The next generation experiment Hyper-Kamiokande [1] builds on the successful strategies used to study neutrino oscillations in the Super-Kamiokande, K2K and T2K experiments, with improvements designed to study the three questions presented above. First, larger detectors with improved photsensors will be used to increase statistics. The baseline design includes two $60 \mathrm{~m}$ height, $74 \mathrm{~m}$ diameter cylindric detectors each corresponding to a fiducial volume of $190 \mathrm{ktons}$. The two detectors will be built either in a staging approach, or in different locations. For the accelerator neutrino beam part of the experiment, a more intense beam will be used to increase statistics, and control of the systematic uncertainties will be improved by an upgrade of the current T2K near detectors [2], and the construction of an additional intermediate water Cerenkov detector (E61). In this paper, we will present the potential of this project for the study of neutrino oscillations, first with only one detector, and then with the different options considered for the second detector.

\section{Long baseline oscillations: Tokai to Hyper-Kamiokande (T2HK)}

\subsection{Experimental apparatus}

As the candidate site for the first Hyper-Kamiokande detector is located $8 \mathrm{~km}$ south of the current Super-K detector, the accelerator neutrino part of the experiment is very similar to the setup of the current T2K experiment [4]. An intense muon neutrino/anti-neutrino beam is produced from accelerated protons hitting a graphite target in J-PARC on the east cost of Japan, and propagates over $295 \mathrm{~km}$ before reaching the far detector located in a direction making a $2.5^{\circ}$ angle with the axis of the beam. A complex of near detectors located $280 \mathrm{~m}$ from the target allows to monitor the neutrino beam, and to study it to control the systematic uncertainties. The main difference with the T2K setup is the presence of an additional intermediate water Cerenkov detector located between $700 \mathrm{~m}$ and $2 \mathrm{~km}$ from the target, which will allow further reduction of the systematic uncertainties by sampling the beam at different off-axis angles [3].

\subsection{Sensitivity study method}

Since the experimental apparatus of the T2HK project is similar to the T2K one, tools developed to study the T2K sensitivity [5] were used to study the physics potential of this project. In this part, we consider one 187 fiducial volume detector, using scaled simulation and reconstruction from the current Super-K detector (in particular improvements coming from the better photo-sensors are 
not considered). A running time of 10 years with a $1.3 \mathrm{MW}$ beam is assumed, with a 1:3 ratio between neutrino and anti-neutrino running modes. Importantly, the mass hierarchy is assumed to be known and to be normal in this part, and the true values of the oscillation parameters considered $\operatorname{are}^{2} \sin ^{2}\left(2 \theta_{13}\right)=0.1, \sin ^{2}\left(\theta_{23}\right)=0.5, \sin ^{2}\left(2 \theta_{12}\right)=0.8704, \Delta m_{32}^{2}=2.4 \times 10^{-3} \mathrm{eV}^{2} / \mathrm{c}^{4}$ and $\Delta m_{21}^{2}=7.6 \times 10^{-5} \mathrm{eV}^{2} / \mathrm{c}^{4}$. The systematic uncertainties are estimated based on the $\mathrm{T} 2 \mathrm{~K}$ experience and the expected improvements coming from the new near and intermediate detectors, as well as the larger atmospheric neutrino control sample for the far detector. The size of those uncertainties are summarized in table 1 .

\begin{tabular}{lccccc}
\hline \hline Mode & sample & $\begin{array}{c}\text { Flux + ND constrained } \\
\text { interaction }\end{array}$ & $\begin{array}{c}\text { ND independent } \\
\text { interaction }\end{array}$ & Far detector & Total \\
\hline$v$ & appearance & $3.0 \%$ & $0.5 \%$ & $0.7 \%$ & $3.2 \%$ \\
$v$ & disappearance & $3.3 \%$ & $0.9 \%$ & $1.0 \%$ & $3.6 \%$ \\
$\bar{v}$ & appearance & $3.2 \%$ & $1.5 \%$ & $1.5 \%$ & $3.9 \%$ \\
$\bar{v}$ & disappearance & $3.3 \%$ & $0.9 \%$ & $1.1 \%$ & $3.6 \%$ \\
\hline \hline
\end{tabular}

Table 1: Systematic uncertainties assumed for the accelerator neutrino sensitivity studies.

\subsection{Expected sensitivities}

For the appearance samples, corresponding to the $v_{e} / \bar{v}_{e}$-like candidate events at the far detector, more than 1000 events are expected in each running mode, with clear differences in the number of events and reconstructed energy spectra predicted for the different possible values of $\delta_{C P}$. This will allow to exclude the conservation of CP symmetry in neutrino oscillations with $5 \sigma$ (resp. $3 \sigma$ ) significance for $57 \%$ (resp. $74 \%$ ) of the possible true values of $\delta_{C P}$, and to measure the value of this parameter with a precision ranging from $7^{\circ}$ (true $\delta_{C P}=0^{\circ}$ ) to $23^{\circ}$ (true $\delta_{C P}=90^{\circ}$ ) as can be seen on figure 1.
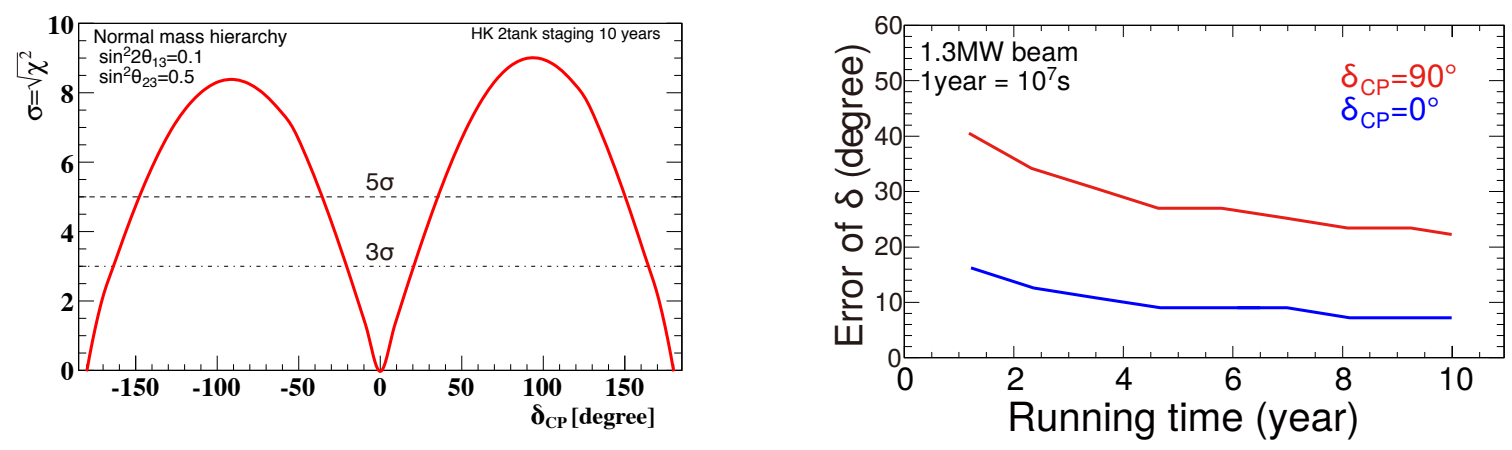

Figure 1: Ability of T2HK to exclude conservation of CP symmetry as a function of the true value of $\delta_{C P}$ (left) and precision of the measurement of $\delta_{C P}$ as a function of exposure (right).

For the disappearance samples ( $v_{\mu}$ and $\bar{v}_{\mu}$-like candidate events), more than 10000 events in each running mode are expected, allowing a precise measurement of the parameters $\sin ^{2}\left(\theta_{23}\right)$ and $\Delta m_{32}^{2}$. Using the measurement of $\sin ^{2}\left(2 \theta_{13}\right)$ by the reactor experiments (assumed to be $\sin ^{2}\left(2 \theta_{13}\right)=$ 
$0.1 \pm 0.005$ ), the precision on $\sin ^{2}\left(\theta_{23}\right)$ ranges from 0.006 (true $\sin ^{2}\left(\theta_{23}\right)=0.45$ ) to 0.017 (true $\left.\sin ^{2}\left(\theta_{23}\right)=0.5\right)$, while the expected precision on $\Delta m_{32}^{2}$ is $1.4 \times 10^{-5} \mathrm{eV}^{2} / \mathrm{c}^{4}$. The octant of $\theta_{23}$ can also be determined with $3 \sigma$ significance for the most favorable values of $\sin ^{2}\left(\theta_{23}\right)$ (fig 2).

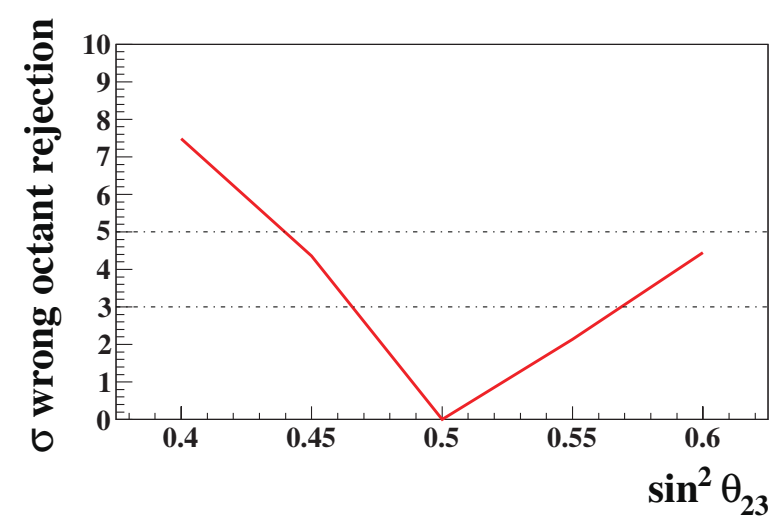

Figure 2: Ability of T2HK to determine the octant of $\theta_{23}$ as a function of the true value of $\sin ^{2}\left(\theta_{23}\right)$.

\section{Atmospheric neutrino oscillations}

Atmospheric neutrinos are produced by the collisions of cosmic rays with particles in the atmosphere. Those collisions produce an isotropic flux of $v_{\mu}, \bar{v}_{\mu}, v_{e}$ and $\bar{v}_{e}$ around the Earth, with a wide range of energies (from $100 \mathrm{MeV}$ to several $\mathrm{TeV}$ ), and which will reach a detector located on the Earth surface after propagating over different distances, from $10 \mathrm{~km}$ to more than $10000 \mathrm{~km}$. This makes those neutrinos very interesting to study neutrino oscillations, and particularly the mass hierarchy. The propagation through matter modifies the neutrino oscillation probability compared to vacuum oscillation, in ways that depend of the mass hierarchy. In particular for a certain combination of neutrino energy and propagation length there is a matter induced resonance in the oscillation probability from the electron to the muon flavor, which happens only for neutrinos if the mass hierarchy is normal, and only for anti-neutrinos if it is inverted.

\subsection{Expected Sensitivities with atmospheric neutrinos only}

Atmospheric neutrino oscillations can be studied in Hyper-Kamiokande in a similar way to what is done in the Super-K experiment [6], and the sensitivity was studied using scaled simulation and analysis tools from Super-K. In particular no improvements of the systematic uncertainties compared to the current Super-K analysis are assumed. The exposure considered corresponds to 10 years of running with a 187 kton fiducial volume detector, and the mass hierarchy is no longer assumed to be known in this part.

Using atmospheric neutrinos only, Hyper-Kamiokande can determine the mass hierarchy with $3 \sigma$ significance for a number of possible true values of $\sin ^{2}\left(\theta_{23}\right)$ if the hierarchy is normal. The sensitivity is lower if the hierarchy is inverted, but $3 \sigma$ significance can still be reached for the largest values of $\sin ^{2}\left(\theta_{23}\right)$. Atmospheric neutrinos also have some sensitivity to the octant of $\theta_{23}$, 
but less than the beam neutrinos. As can be seen on figure 3, in both cases the sensitivity is highly dependent on the true value of $\sin ^{2}\left(\theta_{23}\right)$.
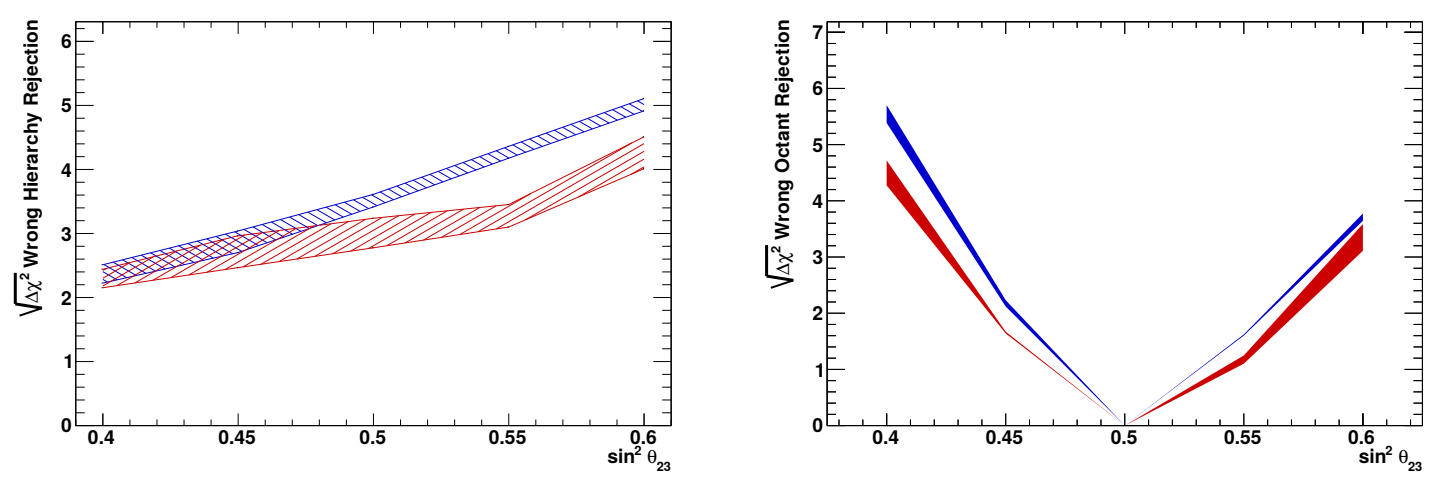

Figure 3: Ability of Hyper-Kamiokande to determine the mass hierarchy (left) and the octant of $\theta_{23}$ (right) using atmospheric neutrinos only. In both figures, blue (red) corresponds to the true hierarchy being normal (inverted), and the width of the bands corresponds to the uncertainty on the true value of $\delta_{C P}$.

\subsection{Expected Sensitivities with beam and atmospheric neutrinos combined}

Atmospheric neutrinos are sensitive to the mass hierarchy, but the precision of the measurement depends on the uncertainty on $\sin ^{2}\left(\theta_{23}\right)$, which is not well constrained by the study of the oscillations of atmospheric neutrinos alone. On the other hand, the beam neutrinos from the J-PARC accelerator allow to measure precisely this parameter, but do not have much sensitivity to the mass hierarchy, mainly due to the too short baseline between J-PARC and Kamioka. With a combined analysis of the oscillations of the two types of neutrinos, the experiment becomes more sensitive to the mass hierarchy. As can be seen on figure 4, the mass hierarchy can then be determined at better than $3 \sigma$ significance for all values of $\sin ^{2}\left(\theta_{23}\right)$, and with $5 \sigma$ significance for the largest values of $\sin ^{2}\left(\theta_{23}\right)$ regardless of whether the true hierarchy is normal or inverted. This combination is also interesting for the search for the violation of CP symmetry: although most of the sensitivity to $\delta_{C P}$ comes from beam neutrinos, there can be degeneracy between the mass hierarchy and the value of $\delta_{C P}$ for certain combinations of true values of the two. In those cases, the atmospheric neutrinos allow to break the degeneracy, and increase the ability to reject the conservation of CP symmetry.

\section{Second detector}

\subsection{Staged approach}

The first option for the second detector is to follow a staged approach, where a second similar detector would be built close to the first one, and start taking data 6 years after the first detector. This would allow to increase the statistics, improving the ability of the experiment to study the violation of CP symmetry and the mass hierarchy. Using beam neutrinos only, the fraction of the possible true values of $\delta_{C P}$ for which conservation of CP symmetry can be excluded with $5 \sigma$ (resp. $3 \sigma$ ) significance would reach $62 \%$ (resp. $78 \%$ ). In combination with atmospheric neutrinos, the mass hierarchy could be determined at better than $4 \sigma$ significance for all true values of $\sin ^{2}\left(\theta_{23}\right)$. 


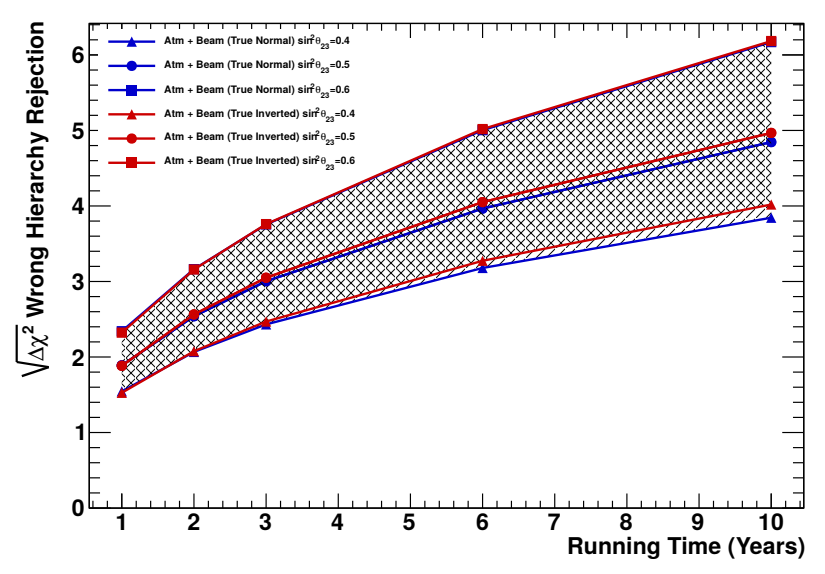

Figure 4: Ability of Hyper-Kamiokande to determine the mass hierarchy using both atmospheric and accelerator neutrinos as a function of running time. Blue (red) corresponds to the true hierarchy being normal (inverted).

\subsection{Detector in Korea}

The other option studied is to build the second detector in Korea [7], as early as possible. This would increase the statistics for atmospheric neutrinos in a very similar way to what a second detector in Kamioka would do, but opens new possibilities for the accelerator neutrino program. As the baseline from J-PARC to Korea is much longer than the one to Kamioka (1000-1100km compared to $295 \mathrm{~km}$ ), the beam neutrinos would propagate through more matter, and therefore their oscillations would become sensitive to the mass hierarchy. The disadvantage of a longer baseline is that the expected number of events is smaller, but this configuration would also benefit the study of CP symmetry: the Korean detector would be located close to the second oscillation maximum, for which the differences between the predicted spectra for different true values of $\delta_{C P}$ are larger than for the first oscillation maximum studied with a detector in Kamioka. Those larger differences would make the experiment less sensitive to systematic uncertainties on the predicted ratio of neutrino and anti-neutrino events.

The analysis used to study the sensitivity of the experiment with a second detector in Korea is slightly different for the beam neutrino part than the one used for the results reported so far, as a result sensitivities for similar configurations might look different in this part. The model used here is based on a more recent T2K analysis (2016), with a systematic uncertainty model focused on the uncertainties likely to affect the study of $\mathrm{CP}$ violation in a large statistics experiment. The sensitivity of the different configurations of the experiment obtained with this analysis for the mass hierarchy and the octant of $\theta_{23}$ can be seen on figure 5. With a second detector in Korea, the mass hierarchy can be determined at more than $6 \sigma$ significance for all possible true values of $\sin ^{2}\left(\theta_{23}\right)$. For the octant determination, the sensitivity is mainly increased by having a second detector, regardless of whether it is located in Korea or in Japan. For the study of CP symmetry, a second detector in Korea allows to break the potential degeneracy between the mass hierarchy and the value of $\delta_{C P}$, similarly to what can be done with atmospheric neutrinos, but with beam 
neutrinos alone. Also a detector in Korea at a smaller off-axis angle, would increase the precision of the measurement $\delta_{C P}$ if the true value of this parameter is close to $\pm \pi / 2$.
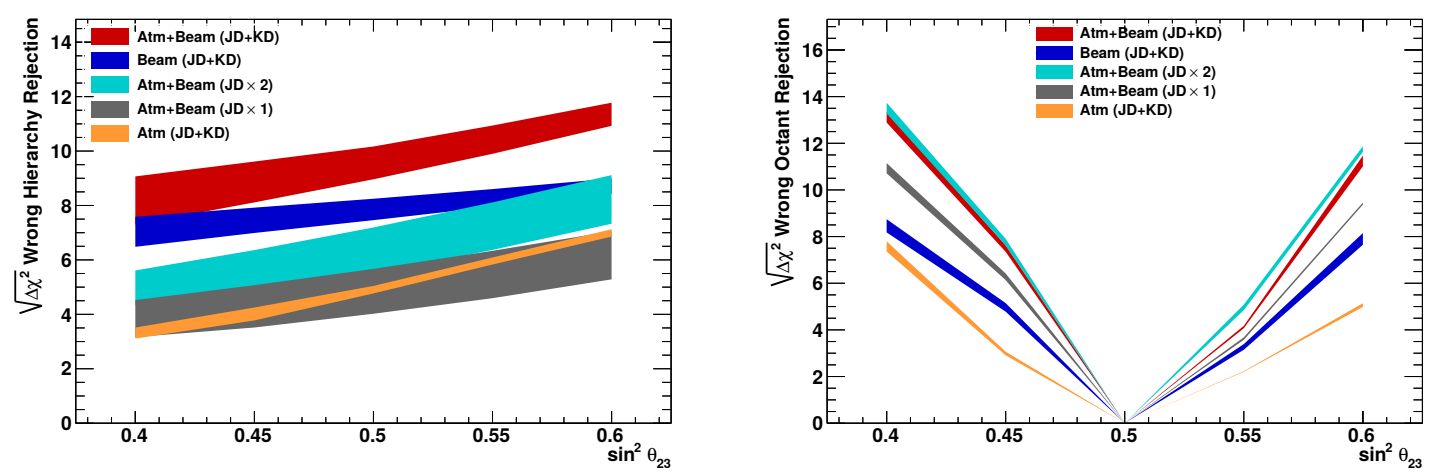

Figure 5: Sensitivity of different configurations of the Hyper-K experiment to the mass hierarchy (left) and the octant of $\theta_{23}$ (right). The width of the bands corresponds to the uncertainty on the true value of $\delta_{C P}$. JD $\equiv$ detector in Japan and $\mathrm{KD} \equiv$ detector in Korea.

\section{Summary}

The next generation neutrino experiment Hyper-Kamiokande will allow to study precisely whether CP symmetry is conserved or not in neutrino oscillations, the neutrino mass hierarchy and the octant of $\theta_{23}$. With a 187 kton fiducial mass detector and combining atmospheric and beam neutrinos, the experiment can exclude the conservation of CP symmetry with $5 \sigma$ significance for $57 \%$ of the possible true values of $\delta_{C P}$ and determine the mass hierarchy with more than $3 \sigma$ significance. A second detector of identical fiducial mass, located either close to the first one (giving a $295 \mathrm{~km}$ baseline for accelerator neutrinos) or in Korea (giving a longer baseline for accelerator neutrinos, around $1000 \mathrm{~km}$ ) would increase the sensitivity. In particular, with a second detector located in Korea, the experiment would be able to determine the mass hierarchy with more than $6 \sigma$ significance.

\section{References}

[1] K. Abe et al. (Hyper-Kamiokande Proto-Collaboration), Hyper-Kamiokande Design Report, KEK-PREPRINT-2016-21, ICRR-REPORT-701-2016-1

[2] A. Blondel, M. Zito et al. CERN SPSC-EOI-015 (2017)

[3] S. Bhadra et al., Proposal for the NuPRISM Experiment in the J-PARC Neutrino Beamline, http://j-parc.jp/researcher/Hadron/en/pac_1607/pdf/P61_2016-17.pdf

[4] K. Abe et al. (T2K collaboration), Nucl. Instrum. Meth., A659,106 (2011)

[5] K. Abe et al. (T2K collaboration), Prog. Theor. Exp. Phys. (2015) 043C01

[6] K. Abe et al. (Super-Kamiokande Collaboration), arXiv:1710.09126 [hep-ex]

[7] K. Abe et al. (Hyper-Kamiokande Proto-Collaboration), arXiv:1611.06118 [hep-ex] 